\title{
Beyond initiation-limited translational bursting: the effects of burst size distributions on the stability of gene expression
}

Hiroyuki Kuwahara ${ }^{1,2}$, Stefan T. Arold ${ }^{1,3}$, and Xin Gao ${ }^{1,2}$

${ }^{1}$ Computational Bioscience Research Center, King Abdullah University of Science and

Technology, Thuwal, 23955, Saudi Arabia

${ }^{2}$ Computer, Electrical and Mathematical Sciences and Engineering Division, King Abdullah

University of Science and Technology, Thuwal, 23955, Saudi Arabia

${ }^{3}$ Biological and Environmental Science and Engineering Division, King Abdullah University of Science and Technology, Thuwal, 23955, Saudi Arabia

\section{Keywords}

gene expression noise; translational bursting; gene regulation; stability control 


\section{Abstract}

A main source of gene expression noise in prokaryotes is translational bursting. It arises from efficient translation of mRNAs with low copy numbers, which makes the production of protein copies highly variable and pulsatile. To obtain analytical solutions, previous models to capture this noise source had to assume translation to be initiation-limited, representing the burst size by a specific type of a long-tail distribution. However, there is increasing evidence suggesting that the initiation is not the rate-limiting step in certain settings, for example, under stress conditions. Here, to overcome the limitations imposed by the initiation-limited assumption, we present a new analytical approach that can evaluate biological consequences of the protein burst size with a general distribution. Since our new model can capture the contribution of other factors to the translational noise, it can be used to analyze the effects of gene expression noise in more general settings. We used this new model to analytically analyze the connection between the burst size and the stability of gene expression processes in various settings. We found that the burst size with different distributions can lead to quantitatively and qualitatively different stability characteristics of protein abundance and can have non-intuitive effects. By allowing analysis of how the stability of gene expression processes changes based on various distributions of translational noise, our analytical approach is expected to enable deeper insights into the control of cell fate decision-making, the evolution of cryptic genetic variations, and fine-tuning of gene circuits. 


\section{Introduction}

The recent advent of single-molecule measurement methods enabled the observation of highly stochastic gene expression in a wide range of organisms [1-6]. Gene expression noise has been shown to have effects reaching far beyond the single protein level and play an important role in evolution and function of biological systems [7-11]. Notably, gene expression noise is key to stochastic switching, which enables a clonal population of cells to have phenotypic variability in a homogeneous environment. Thus, the accurate characterization of gene expression noise and its effects is important to understand diverse biological phenomena ranging from microbial pathogenicity $[12,13]$ to cancer cell heterogeneity $[14,15]$.

In prokaryotes, a major source of gene expression noise is reported to be translational bursting. Highly variable and skewed bursts in protein synthesis arise from efficient translation of lowcopy-number mRNAs, and they contribute significantly to the protein expression noise $[16,17]$. To gain analytical and quantitative insights into the effects of translational bursting on protein distributions and cellular phenotypes, previous studies relied on an assumption that the ratelimiting step of the mRNA translation process be the initiation $[18,19]$. Consequently, all of the control steps in the translational process, including initiation, elongation and termination (Fig. 1), are approximated by only a single reaction. This assumption is the theoretical basis to approximate the protein burst size by a geometric distribution, which, in turn, yields an analytical solution of the stationary protein expression distribution (i.e., the distribution for the long-term frequency of the protein copy number). Although this assumption was supported experimentally for the expression of many E. coli genes under a specific non-stress condition $[2,20]$, a number of studies have reported that other factors in protein synthesis such as those involved in translation elongation - including codon usage bias and ribosomal pauses - play significant roles in determining translational efficiency [21-25]. Thus, despite the fact that the most commonly held view of translation is the initiation-limited process in most endogenous genes [26,27], these results show that initiation is not the sole determinant for translation rate in a wide range of experimental settings. This, in turn, strongly suggests that other factors can play an crucial role in determining the protein burst size. Indeed, recent theoretical studies showed evidence that translation elongation can have strong effects on translational bursting as well as protein abundance [28-30]. An inclusion of any additional effects on translational noise is, however, not possible with models with a single-step translation process. 
Here, we developed an analytical model in which we can overcome the limitation of the assumption that the translational initiation is the rate-limiting reaction. This approach allowed us to study the effects of translational noise on the protein copy number distribution in a more general setting. By accommodating situations with arbitrarily distributed burst sizes, our approach effectively captures all intrinsic and extrinsic factors which can shape the protein burst size, including the initiation of translation, the translocation of ribosome, and the variability in the level of ribosome and charged tRNAs within isogenic cells. Whereas the analytical expression of the protein distribution was seen to be difficult with arbitrarily distributed burst sizes [31], we were able to gain insights into the effects of the the protein synthesis noise by focusing on the stability characteristics of gene expression processes.

\section{Model}

We consider the regulation of a single gene depicted in Fig. 1, in which transcription factor $\mathrm{S}$ controls the rate of the synthesis of mRNA M, which is translated into protein X. A detailed description of our gene expression model is given in the Methods section. Unlike previous analytical gene expression models which make with the initiation-limited translation assumption to impose the protein burst size $V$ to follow a geometric distribution, our new model considers $V$ to follow an arbitrary distribution. The only constraint we impose on $V$ is that its first two moments, $\mu_{1}$ and $\mu_{2}$, exist and are finite, which must be always valid under any finite-volume environments. That is, our model is more general and works with any shape of the protein burst size distribution provided that its average and variance are given. Our analytical model is based on a continuous stochastic process governed by the following Fokker-Planck equation:

$$
\begin{aligned}
\frac{\partial p(x, t \mid s)}{\partial t}= & -\frac{\partial}{\partial x}[A(x \mid s) p(x, t \mid s)] \\
& +\frac{1}{2} \frac{\partial^{2}}{\partial x^{2}}[D(x \mid s) p(x, t \mid s)]
\end{aligned}
$$

where $p(x, t \mid s)$ is the probability that the copy number of protein $\mathrm{X}$ is $x$ at time $t$ given that the copy number of transcription factor $\mathrm{S}$ is $s, A(x \mid s)$ is the deterministic term of the process called the drift function, and $D(x \mid s)$ is the noise term called the diffusion function (see Methods for the derivation). Here, $A(x \mid s)=\mu_{1} q(s)-k_{d e g} x$ and $D(x \mid s)=\mu_{2} q(s)+k_{\text {deg }} x$ where $q(s)$ is a thermodynamic-based model for transcription kinetics (see Methods for the detail). This model contains biologically relevant kinetic parameters, $k_{b}, k_{a}, K_{d}$, and $n$, where $k_{b}$ is the 
basal transcription rate, $k_{a}$ is the activated transcription rate, $K_{d}$ is the dissociation constant, and $n$ describes binding cooperativity. Let $X_{\infty}$ be a random variable with its density function being $p_{s}(x \mid s)=\lim _{t \rightarrow \infty} p(x, t \mid s)$, which describes the stationary, long-term expression level of protein X. Then, following Gillespie [32], we define a stable state of the protein level, $x_{s s}$, to be a position at which $p_{s}(x \mid s)$ has a local maximum (i.e., a peak of the stationary protein expression distribution). Correspondingly, we define an unstable state of the protein level, $x_{u s}$, to be a position at which $p_{s}(x \mid s)$ has a local minimum (i.e., a bottom of the stationary protein expression distribution). Biologically, a stable state is an abundance level to which the copy number of protein $\mathrm{X}$ is eventually attracted, while an unstable state is an abundance level from which the copy number of protein $\mathrm{X}$ is seen to move away. To analyze the stability characteristics of the protein expression process, we define a function based on $A(x \mid s)$ and $D^{\prime}(x \mid s)$ :

$$
\alpha(x \mid s)=2 A(x \mid s)-D^{\prime}(x \mid s)
$$

Using this function, we can express the sufficient condition for a stable state, $x_{s s}$, and an unstable state, $x_{u s}$, such that $x_{s s}$ satisfies $\alpha\left(x_{s s} \mid s\right)=0$ and $\alpha^{\prime}\left(x_{s s} \mid s\right)<0$, while $x_{u s}$ satisfies $\alpha\left(x_{u s} \mid s\right)=0$ and $\alpha^{\prime}\left(x_{u s} \mid s\right)>0$ (see Supplementary Material Section S3).

\section{Results}

\section{Gene regulation with a constitutive promoter}

First, we consider the simplest case in which a gene is expressed from a constitutive promoter, instead of a regulated promoter. Many constitutive promoters in E. coli are those that require the housekeeping sigma factor RpoD to direct RNA polymerase to form a close complex and initiate transcription of essential genes in the growth phase [33]. To model the constitutive expression of a gene, we set the transcriptional reaction to have a fixed rate: $k_{\text {prod }}=q(S)$. In this setting, if the burst size, $V$, is geometrically distributed - as required when imposing translation as an initiation-limited process - then $p_{s}(x)$ is reported to be well approximated by a gamma distribution with scale parameter being $\mu_{1}$ and shape parameter being $k_{\text {prod }} / k_{d e g}[19]$. Since $\mu_{1}$ is the average protein copy number synthesized from a single mRNA molecule and $k_{\text {prod }} / k_{\text {deg }}$ describes the average number of the mRNA synthesis events with respect to the average protein lifetime, we can relate the protein distribution with biophysical parameters for 
the protein production. While we confirmed that the gamma distribution can well approximate the protein distribution in this setting, we also found that, when the burst size distribution, $p_{V}(v)$, deviates from a geometric distribution, the quality of the approximation by a gamma distribution can decrease (Fig. 2).

Even when $V$ is not a geometric random variable, that is, when the initiation-limiting assumption does not hold, we can still use our model to analytically determine the condition of stable protein levels. In fact, by examining properties of the function $\alpha(x)$ (see Supplementary Material Section S6), we found that, when $2 \mu_{1} k_{\text {prod }}>k_{d e g}$, we have a monostable system with a poisitve stable state

$$
x_{s s}=\mu_{1} \frac{k_{\text {prod }}}{k_{\text {deg }}}-\frac{1}{2} .
$$

This expression shows that the stable protein level $x_{s s}$ is independent of the second moment of the protein burst size $\mu_{2}$. That is, if the average burst size is fixed, the stable protein expression level is not affected by changes in the variance of the burst size and, more generally, in the shape of the burst size distribution. Also, this shows that the stochastic prediction of the stable protein level is always lower than the deterministic counterpart by $1 / 2$, suggesting that gene expression noise decreases the stable state of the protein level.

To quantify the stability of the protein copy number around a stable state $x_{s s}$, we made a Gaussian assumption to obtain the expression for a "stability width" $w$ (see Supplementary Material Section S5) as

$$
w=\sqrt{\frac{1}{2} \frac{k_{\text {prod }}}{k_{\text {deg }}}\left(\mu_{2}+\mu_{1}\right)-\frac{1}{4}} .
$$

Interestingly, this shows that, since $\mu_{2} \geq \mu_{1}^{2}, \mu_{2}$ becomes dominant over $\mu_{1}$ as $\mu_{1}$ increases, implying that, as the average protein level increases, the noise level of the protein becomes less dependent on the average level.

To examine the predictive power of $x_{s s}$ and $w$, we simulated the underlying discretestochastic model governed by the chemical master equation for 10,000 times (see Supplementary Material Section S3) and generated $X_{\infty}$ for various distributions of $V$ (Fig. 3). Our results show that, while the analytical expression of $p_{s}(x)$ may become intractable, these measures can capture its stability characteristics regardless of the setting of the bursting size distribution. This feature allows us to analytically predict how conditions for stable gene expression change even in cases where translation is not seen as an initiation-limited process. 


\section{Gene regulation with a regulated promoter}

Next, we examined a case where the transcription is regulated by a transcription factor $\mathrm{S}$ (Fig. 4a). In this analysis, we assumed that the gene regulation follows the Jacob and Monob model [34]; that is, $\mathrm{S}$ is a transcriptional repressor, which, upon binding to the operator site, prevents the RNA polymerase from initiating transcription (i.e., $k_{a}=0$ ). Since most of bacterial transcription factors are homodimers (e.g., helix-turn-helix dimers) that bind to two inverted repeat half-sites [35], we considered S to be a homodimeric transcriptional repressor. We further consider a general case where the repressor level, $S(t)$, is independent of the protein level, $X(t)$, and the time-scale of the repressor is fast. In this setting, we analyzed how changes in the variances of the repressor level and the protein burst size affect the stability of the protein level (Fig. 4a). Since $S(t)$ is seen to be independent and identically distributed at each time point in the eye of the transcription reaction, we have $A(x)=\mu_{1}\langle q(S)\rangle-k_{d e g} x$ and $D^{\prime}(x)=k_{d e g}$, yielding

$$
x_{s s}=\mu_{1} \frac{\langle q(S)\rangle}{k_{d e g}}-\frac{1}{2} .
$$

This shows that $x_{s s}$ does not depend on the second moment of the burst size, but does depend on the average transcription rate, $\langle q(S)\rangle$. That is, similar to the gene expression with constitutive promoter, the stable protein expression level is not affected by the shape of the burst size distribution as long as the average burst size is fixed. Since we have a homodimer repressor in our model, binding cooperativity in the transcription reaction kinetic is set to $n=2$. In this setting, transcriptional repression is activated (i.e., transcription rate function $q(s)$ curves upward) when the abundance level of repressor $\mathrm{S}$ is greater than $K_{d} / \sqrt{3}$, which is called the inflection point at which the second derivative of $q(s)$ has the value of zero. In this convex regime, Jensen's inequality states that $\langle q(S)\rangle>q(\langle S\rangle)[18]$, that is, the average of the function is greater than the function of the average. This difference can become larger as the variance of $S$ becomes larger (Fig. 4b). Thus, our analytical model predicted that the stable protein copy number can increase as the variance of the repressor level increases.

To test this hypothesis, we simulated the underlying discrete-stochastic model (see Supplementary Material Section S3). We ran simulations for 10,000 times each with three different values of the average repressor level, $E[S]$, in each of which we had three different settings for the variance of $S$, and two different settings for $\mu_{2}$. We observed that stable protein expression level obtained from the simulation closely agree with those predicted by Eq. 5, indicating that 
our analytical model is accurate and $x_{s s}$ is indeed independent of $\mu_{2}$ (Fig. 4c). Our simulation also showed that the relation between the stable protein level and the signal fluctuations is context dependent; while the stable protein level appears to be independent of the variance of the repressor level when the average repressor level is close to the inflection point (i.e., $E[S]=25$ in Fig. 4), the stable protein level can become substantially higher as the variance of the repressor increases when the repressor is activated (i.e., $E[S]=50$ and $E[S]=100$ in Fig. 4).

Next, we predicted how the fluctuations of the repressor level and the burst size affect the noise level of the protein copy number, which was measured by the coefficient of variation, $c v\left(X_{\infty}\right)=\sigma\left(X_{\infty}\right) /\left\langle X_{\infty}\right\rangle$. Whereas the analytical expression of $c v\left(X_{\infty}\right)$ is not feasible for an arbitrarily distributed $V$, it may be possible to well approximate it by the ratio of the stability width to the stable state, which is given by

$$
\frac{w}{x_{s s}}=\frac{\sqrt{\frac{1}{2} \frac{\langle q(S)\rangle}{k_{d e g}}\left(\mu_{2}+\mu_{1}\right)-\frac{1}{4}}}{\mu_{1} \frac{\langle q(S)\rangle}{k_{d e g}}-\frac{1}{2}} .
$$

Thus, we used this expression to predict the effects of the fluctuations of $S$ and $V$ on $\operatorname{cv}\left(X_{\infty}\right)$. Eq. 6 predicts that an increase in the variance of the burst size results in higher level of protein expression noise. It also predicts that the average of $q(S)$ has an inverse relation with $c v\left(X_{\infty}\right)$. That is, it predicts that an increase in the average transcription rate reduces the noise level of the protein expression. Since higher fluctuations in $S$ in the convex regime increase the value of $\langle q(S)\rangle$, it may be possible that an increase in the variance of $S$ can cause $\operatorname{cv}\left(X_{\infty}\right)$ to decrease in the convex regime. To test these analytical predictions, we measured $c v\left(X_{\infty}\right)$ from the simulation data. The results corroborate our analytical predictions (Fig. 4d). In particular, when $E[S]=100$, a higher variance in $S$ markedly resulted in a lower value of $c v\left(X_{\infty}\right)$.

Here, the results show that a high noise level in a homodimer repressor can reduce the noise level of the target protein when the repression effect is relatively high. They also indicate that transcriptional repressors can play a role not just to silence gene expression but also to enhance the stability of protein abundance levels with a noise filtering capability. Indeed, a recent review paper extensively discussed multifaceted roles of transcriptional repressors in the context of developmental processes, including a role as a fine-tuner of gene expression levels [36]. In support of this view, a synthetic biology experiment [37] constructed noise-tuning and -reduction systems in yeast based on transcriptional regulation with a combination of TetR repressor and ATc inducer. These experimental observations provide evidence that transcriptional repressors 
can be used to reduce the protein expression noise. Since the noise-enhanced stability mechanism allows for the reduction of the protein expression noise, it is plausible that gene regulation with transcriptional repressors can exhibit noise-enhanced stability. To experimentally test this prediction, one would need to be able to control the noise level of the repressor and measure the reporter protein at the single-molecule level in each cell. The noise level of the repressor can be controlled by mutating the promoter region and the ribosome binding site of its gene $[16,38,39]$, while the abundance of the reporter protein can be quantified using single-molecule detection techniques for protein expression, for example, based on flow cytometry $[16,38]$ and fluorescence microscopy $[40,41]$.

\section{Gene regulation with positive feedback loop}

The positive-feedback motif is widely used to implement multistability, which is a key ingredient of heritable, epigenetic control mechanisms as it provides a memory mechanism $[42,43]$. Whereas the traditional deterministic framework predicts that a positively cooperative transcription factor is a necessary condition for bistability in feedback-based transcriptional regulation, recent theoretical and experimental studies showed that gene expression noise could induce bistability even with a non-cooperative transcription factor [19,44].

Here, we are interested in studying how, by keeping the average burst size fixed, the variance of the burst size can affect the number of positive stable and unstable states in the system. Since this problem is transformed into the number of sign-changes in $\alpha(x)=2 A(x)-D^{\prime}(x)$ for $x>0$, we examined the shape of $2 A(x)$ and $D^{\prime}(x)$ to analyze the effects of $\mu_{2}$ on the stability of the protein expression process based on the nature of binding cooperativity of the transcriptional activator - that is, based on the value of $n$ (see Supplementary Material Section S5.2).

We first considered the negative cooperativity. (i.e., $n<1$ ). In this setting, we found that there are two different cases for the stability characteristics of the protein expression process (see Supplementary Material Section S6.3):

Case $(i)$ : The process has two attractor states: one at zero and the other one is a higher expression stable state, which are separated by an unstable sate.

Case (ii): The process has no stable states nor unstable state. The protein expression process has an attractor state at value zero.

We found that, by making the variance of the burst size small, we can guarantee the condition 
for Case $(i)$ in a wide range of parameter conditions (Fig. 5a). However, a lower level of burst size variance may make the attraction point at zero unnoticeable (see Supplementary Material Section S6.3). Another analytical observation is that an increase in the variance of the burst size can change the stability characteristics of the system from Case $(i)$ to Case $(i i)$ by vanishing the higher expression stable state (more detailed discussion in Supplementary Material Section S6.3). This suggests a potentially important role of the burst size distribution in the control of the stability characteristics of gene expression processes.

We next considered the non-cooperative setting (i.e., $n=1$ ). In this setting, we can express the sufficient condition for the system to have monostablility as follows (see Supplementary Material Section S6.4 for detail):

$$
\mu_{2}<K_{d}\left(2 \mu_{1} k_{b}-k_{d e g}\right) /\left(k_{a}-k_{b}\right) .
$$

That is, when Condition 7 is satisfied, the protein expression distribution, $p_{s}(x)$, has a single peak at $x=x_{s s}$. By increasing the burst size variance, $D^{\prime}(x)$ increases, and we can eventually introduce a valley to $p_{s}(x)$ with its bottom at $x=x_{u s}$, separating the higher expression stable state $x_{s s}$ and an attraction point at zero (Fig. $5 \mathrm{~b}$ ). As the variance is increased even further, the stable state will eventually become vanished, making $p_{s}(x)$ a monotonically decreasing function with the peak at 0. Fig. 6 illustrates an instance where an increase in the burst size variance can result in different stability characteristics; that is, Poissonian translational noise (i.e., burst size with the variance at the same level as the average) leads to a single positive stable state, while geometric translational noise (i.e., burst size with the standard deviation at the same level as the average) leads to one unstable state surrounded by two peaks at zero and a positive stable state. This shows that changes in the noise level of the burst size can lead to qualitative changes in the shape of the protein expression distribution, suggesting an important role of the burst size distribution in the control of the protein abundance.

Based on the results from the negative- and non-cooperativity settings, our model predicts that, if $n \leq 1$, the manipulation of the variance of the burst size alone cannot give rise to two positive stable states, while bistability involving a point of attraction at $x=0$ is possible. This agrees with previous studies $[19,31]$ in that a non-cooperative feedback system with a memoryless burst size distribution can result in bistability with a point of attraction at value zero. Further, since our new approach considers an arbitrarily distributed burst size, we were 
able to find that, in some cases, it is impossible for a stochastic process to exhibit specific stability characteristics predicted by the corresponding deterministic process. This suggests that gene expression noise from translational bursting can substantially constrain the qualitative behavior of gene expression processes.

The last one to be considered in the positive feedback loop is the positive cooperativity setting (i.e., $n>1$ ). In this setting, the stability characteristics can be widely different between a stochastic gene expression process and its deterministic counterpart. In the deterministic context, the process is bistable, only if the drift function $A(x)$ is negative at the local minimum point and it is positive at the local maximum point. In the stochastic context, on the other hand, the process can also exhibit bistability under other conditions because of the nonlinear noise term $D(x)$ (Supplementary Material Section S6.5). In addition, by manipulating the burst size variance, we found that the number of positive stable states in the process can change (Supplementary Material Section S6.5). Fig. 5c, for example, shows that, while a feedback process with a geometrically distributed protein burst size (i.e., with the initiation-limited translation assumption) exhibits monostablility, lowering of burst size variance can make the process bistable.

To and Maheshri showed that gene expression noise can induce a bimodal protein distribution in a synthetic positive feedback gene circuit in yeast [44]. Our results predict that, depending on the stochasticity level, the shape of the protein distribution can qualitatively change and, in particular, that higher gene expression noise from protein burst can also transform a bimodal protein distribution to a unimodal one. Furthermore, Fig. 5c shows that, while the corresponding deterministic process predicts monostability with a higher expression stable state, the stochastic process with any shape of the burst size distribution is not capable of displaying the same stability characteristic. This suggests that the stochasticity in a gene expression process can restrict the repertoire of stability characteristics.

\section{Gene expression noise reduction based on higher variance in protein burst size}

Regardless of the nature of the binding cooperativity in positive autoregulation, an increase in the variance of the burt size causes $D^{\prime}(x)$ to move upwards since $D^{\prime}(x)$ is a linear function of $\mu_{2}$, resulting in the shifting of a higher expression stable state to a lower level and the unstable state to a higher level (Fig. 5). In a case of systems with two positive stable states, 
the lower expression stable state is also lowered with the upward shift in $D^{\prime}(x)$ (Fig. 5c). These results predict that an increase in the burst size variance can reduce the attraction domain of the higher-expression state while enhancing the stability of the lower-expression stable state, which, depending on the size of changes, can shift the mean and the variance of $X_{\infty}$ to lower levels. This suggests that a higher burst size variance can decrease the average protein level and it can also decrease the variance of the protein level. Indeed, our model shows that it is possible to induce the stability of the protein level with a higher noise level in the burst size (Fig. 7). As predicted, this striking phenomenon was accompanied by an decrease in the average protein level. Because of this, the noise level measured by the coefficient of variation can decrease as the variance of the burst size increases.

\section{Gene regulation with negative feedback loop}

We next considered gene regulation with negative feedback loop. Negative feedback loop can act as a noise filter and has been shown to increase the stability of the gene expression process [45]. We first analyzed the shape of $2 A(x)$ and $D^{\prime}(x)$, focusing on analysis of how an increase in the variance of the burst size affects the stable state of the protein level. Regardless of the nature of the binding cooperativity, our model predicts that an increase in the variance of the burst size leads to an increase in the protein expression level (see Supplementary Material Section S6.6).

The effects of the variance in the burst size on the stability characteristics of the negative feedback protein expression process is illustrated in (Fig. 8). By analyzing the number of points at which $2 A(x)$ and $D^{\prime}(x)$ equilibrate in the negative- and non-cooperative cases (i.e., $n \leq 1$ ), we found that the protein expression process has one positive stable state (see Supplementary Material Section S6.6). In the positive-cooperative setting (i.e., $n>1$ ), our analysis shows that, if the value of $2 A(x)$ at the point where the second derivative of $A(x)$ is zero (i.e., at the inflection point) is greater than the degradation constant $k_{d e g}$, then we can guarantee that this gene expression process has a high protein expression level with any setting of the protein burst size distribution (see Supplementary Material Section S6.6).

\section{Discussion}

Through a long process of evolution, organisms have acquired mechanisms to control and exploit inherent randomness so as to increase their fitness in their living environments in a variety 
of ways. While recent advances in high-resolution single-molecule and single-cell experimental techniques have identified high stochasticity in gene expression profiles of a wide range of organisms, it still remains unclear, for example, what consequences can arise from gene expression noise in the function, phenotypes, and fitness of organisms in various environments. Since analytical models can clearly relate gene expression noise to its effects, they bear great promise to substantially enhance our understanding of this biological phenomenon.

Here, we developed a stochastic gene expression model to study the effects of a major source of gene expression noise, namely translational bursting. This analytical model relaxes one of the key assumptions made by previous analytical gene expression models $[18,19,46]$ and treats the protein burst size as an arbitrarily distributed random variable. Although hampering the analytical solution of the stationary protein distribution, this treatment allows us to analytically study how a different distribution of the protein burst size can influence the stability of gene expression processes. With this approach, we predicted non-intuitive effects of changes in the burst size distribution on the stability of gene expression processes. For example, we were able to predict that the protein distribution can change from a bimodal one to a unimodal one as the variance of the protein burst size increases in positive-feedback gene circuits. While previous analytical studies showed noise-induced bistability $[19,31]$ with the initiation-limited translational bursting, our new approach was able to show that stability characteristics can change based on the stochasticity level of the burst size. This includes the architectural feature to exhibit noise-enhanced stability. Although this result may appear counter-intuitive at first glance, noise-enhanced stability has been observed in various physical systems [47-49]. In a range of biological systems - from gene regulatory systems to ecosystems - theoretical predictions of such mechanisms to deal with environmental and intrinsic fluctuations have been discussed [50-52]. In some biomolecular systems, such as those for development and cell fate decisions, the ability to achieve precise spatiotemporal control of gene expression patterns is especially essential. In such systems, various types of gene regulatory architecture, including those with the noise-enhanced stability feature, might have evolved to implement robust noise-filtering mechanisms [53].

Collectively, our findings from this analytical approach strongly suggest that the distribution of the burst size can have profound effects on cellular phenotypes and fitness. Thus, we predict that the burst size distribution may be subject to selection and that organisms may have evolved to fine-tune the burst size distributions of their endogenous proteins by adjusting various 
intrinsic and extrinsic factors involved in protein synthesis, including translation elongation.

A classical example of gene regulatory mechanisms involving translation elongation is attenuation in the E. coli trp operon, in which the normal level of charged tRNA ${ }^{\operatorname{tr} p}$ allows ribosomes to traverse an mRNA strand without interruption, which, in turn, creates a termination hairpin in the mRNA, leading to premature termination of transcription [54]. This mechanism is used to ensure fine-tuned control of the production of tryptophan and to adjust the rate of the production between nutritional stress and normal conditions [55]. Further advances in ribosome profiling methods enabled monitoring of translation elongation at the single-codon resolution under various stress conditions [56]. Using such high-resolution ribosome profiling method, recent study reported that ribosomal translocation plays a crucial role in determining translation rate under amino-acid starvation conditions, during which hidden variations among synonymous codon variants unveil significant differences in elongation rates [25]. These examples provide clear evidence for the importance of additional factors beyond translation initiation, which cannot be captured by previous analytical models. Our model can now be used to understand how changes in elongation rates under various stress conditions correspond to changes in protein burst size distributions and how such changes can influence the stability of gene expression processes as well as the emergence of phenotypic variations.

If the protein burst size of a gene expression process is seen to follow a geometric distribution under any conditions, that is, if the initiation-limited translation process is always assumed to be valid, then our model does not offer any additional advantage over previous methods. However, if the stability of a given gene expression process is observed to change based on its context including environmental stress conditions, then analysis with our analytical model can lead to a deeper understanding of the underlying mechanism. In fact, changing the expression level of genes is crucial part of cellular stress-response, which allows a population of canalized organisms to reveal phenotypic variations in response to environmental stress $[57,58]$. Thus, our analysis may provide unprecedented insights into how multifaceted biological noise are integrated into biological functions in various environmental conditions and how the variation in gene expression noise may affect stress-response mechanisms. Finally, since our analytical predictions can map experimentally-tunable parameters to the shape of the protein distribution, it can provide better design of gene circuits with specific stability requirements. 


\section{Methods}

\section{Gene expression model}

Our kinetic model represents a gene expression process that has four reaction processes: transcription, translation, mRNA degradation, and protein degradation. The transcription rate is described by a thermodynamics-based model, which assumes that the interaction between transcription factors and DNA binding sites is rapid and at equilibrium $[59,60]$. This rapid equilibrium assumption has traditionally been seen to be valid in prokaryotic gene expression $[61,62]$, and thermodynamic-based models have been used in a wide range of biological systems $[63,64]$. With this transcription kinetic model, the reaction time to synthesize one molecule of mRNA $\mathrm{M}$ is given by an exponential distribution with parameter $q(s)=k_{b}[1-w(s)]+k_{a} w(s)$, where $w(s)=s^{n} /\left(K_{d}^{n}+s^{n}\right)$ is the probability that transcription factor $\mathrm{S}$ binds to the operator site, $s$ is the copy number of $\mathrm{S}, k_{b}$ is the basal transcription rate, $k_{a}$ is the activated transcription rate, $K_{d}$ is the dissociation constant, and $n$ describes binding cooperativity. To fine-tune gene expression processes, specific values of these biophysical parameters can be implemented by modifying non-coding regions of DNA [44,65-69]. Protein degradation is modeled using a single-step reaction, and the reaction time to degrade one molecule of protein $\mathrm{X}$ is given by an exponential distribution with parameter $k_{d e g}$. The protein is assumed to be stable, and its half-life is dominated by dilution via cell division, while the mRNA half-life is assumed to be very short. These modeling assumptions are in line with previous analytical models, and they are seen to be biologically plausible $[6,18,19,46]$.

We describe the reaction time to degrade one molecule of mRNA M by an arbitrarily distributed random variable $T_{1}$ and the reaction time to synthesize one molecule of protein $\mathrm{X}$ by an arbitrarily distributed random variable $T_{2}$. Since the reaction time of any single-step elementary reaction necessarily follows an exponential distribution (as seen in transcription and mRNA degradation), the difference between the previous analytical models and ours is the time distributions of mRNA degradation and translation. That is, unlike the previous models, which represent the four reaction processes by single-step elementary reactions, our model is able to describe more complex dynamics of gene expression with $T_{1}$ and $T_{2}$ following general distributions.

This feature allows our model to capture a multi-step process of mRNA degradation [70,71] and to relax the assumption that initiation be the rate-limiting step of translation. 


\section{Derivation of the analytical model}

The probability distribution of the burst size $V, p_{V}(v)$, can be defined based on $T_{1}$ and $T_{2}$ (see Supplementary Material Section S1). For example, if both mRNA degradation and translation are assumed to be based on single-step reactions, then $p_{V}(v)$ becomes a mixture of Poisson distributions with exponentially distributed rates, yielding a geometrically distributed $V$ as reported in previous studies $[18,19,31,46]$. This result is a special case of the Poisson-Gamma mixture, whereby a negative binomial distribution is derived from a mixture of Poisson distributions with Gamma-distributed rates. Although this approximation facilitates the analytical description of translational bursts, it necessarily restricts the biological features that can be captured.

By letting $p(x, t \mid s)$ be the probability that the protein copy number is $x$ at time $t$ given the copy number of transcription factor $\mathrm{S}$ is $s$, we can express a partial differential equation called the chemical master equation, which describes the time-evolution of $p(x, t \mid s)$ (see Supplementary Material Section S2). By making a continuous approximation and resorting to the second-order Taylor expansion, we can simplify the master equation to the Fokker-Planck equation in Eq. 1 (see Supplementary Material Section S2).

\section{Acknowledgments}

Research reported in this paper was supported by competitive research funding from King Abdullah University of Science and Technology (KAUST).

\section{References}

[1] Golding I, Paulsson J, Zawilski S, Cox E (2005) Real-time kinetics of gene activity in individual bacteria. Cell 123: 1025-1036.

[2] Cai L, Friedman N, Xie XS (2006) Stochastic protein expression in individual cells at the single molecule level. Nature 440: 358-362.

[3] Xie XS, Choi PJ, Li GW, Lee NK, Lia G (2008) Single-molecule approach to molecular biology in living bacterial cells. Annu Rev Biophys 37: 417-444.

[4] Raj A, Peskin C, Tranchina D, Vargas D, Tyagi S (2006) Stochastic mRNA synthesis in mammalian cells. PLoS Biol 4: e309. 
[5] Raj A, van Oudenaarden A (2009) Single-molecule approaches to stochastic gene expression. Annual review of biophysics 38: 255-270.

[6] Taniguchi Y, Choi PJ, Li GW, Chen H, Babu M, et al. (2010) Quantifying E. coli proteome and transcriptome with single-molecule sensitivity in single cells. Science 329: 533-538.

[7] Losick R, Desplan C (2008) Stochasticity and cell fate. Science 320: 65.

[8] Raj A, van Oudenaarden A (2008) Nature, nurture, or chance: stochastic gene expression and its consequences. Cell 135: 216-226.

[9] Eldar A, Elowitz MB (2010) Functional roles for noise in genetic circuits. Nature 467: $167-173$.

[10] Johnston RJ Jr, Desplan C (2010) Stochastic mechanisms of cell fate specification that yield random or robust outcomes. Annual Review of Cell and Developmental Biology 26.

[11] Kuwahara H, Soyer OS (2012) Bistability in feedback circuits as a byproduct of evolution of evolvability. Mol Syst Biol 8: 564 .

[12] Kuwahara H, Myers CJ, Samoilov MS (2010) Temperature control of fimbriation circuit switch in uropathogenic Escherichia coli: Quantitative analysis via automated model abstraction. PLoS Comput Biol 6: e1000723.

[13] Jørgensen M, Raaphorst R, Veening JW (2013) Noise and Stochasticity in Gene Expression: A Pathogenic Fate Determinant, s.n., chapter 6. Methods in Microbiology. 40 edition, pp. 157-175. Relation: http://www.rug.nl/research/gbb/ Rights: University of Groningen, Groningen Biomolecular Sciences and Biotechnology Institute.

[14] Pujadas E, Feinberg AP (2012) Regulated noise in the epigenetic landscape of development and disease. Cell 148: 1123-1131.

[15] Landau DA, Clement K, Ziller MJ, Boyle P, Fan J, et al. (2014) Locally disordered methylation forms the basis of intratumor methylome variation in chronic lymphocytic leukemia. Cancer Cell 26: 813-825.

[16] Ozbudak EM, Thattai M, Kurtser I, Grossman AD, van Oudenaarden A (2002) Regulation of noise in the expression of a single gene. Nat Genet 31: 69-73. 
[17] Kærn M, Elston T, Blake W, Collins J (2005) Stochasticity in gene expression: from theories to phenotypes. Nature Reviews Genetics 6: 451-464.

[18] Paulsson J, Berg OG, Ehrenberg M (2000) Stochastic focusing: fluctuation-enhanced sensitivity of intracellular regulation. Proc Natl Acad Sci U S A 97: 7148-7153.

[19] Friedman N, Cai L, Xie XS (2006) Linking stochastic dynamics to population distribution: an analytical framework of gene expression. Phys Rev Lett 97: 168302.

[20] Yu J, Xiao J, Ren X, Lao K, Xie XS (2006) Probing gene expression in live cells, one protein molecule at a time. Science 311: 1600-1603.

[21] Gustafsson C, Govindarajan S, Minshull J (2004) Codon bias and heterologous protein expression. Trends Biotechnol 22: 346-353.

[22] Tuller T, Waldman YY, Kupiec M, Ruppin E (2010) Translation efficiency is determined by both codon bias and folding energy. Proc Natl Acad Sci U S A 107: 3645-3650.

[23] Navon S, Pilpel Y (2011) The role of codon selection in regulation of translation efficiency deduced from synthetic libraries. Genome Biol 12: R12.

[24] Liu T, Kaplan A, Alexander L, Yan S, Wen JD, et al. (2014) Direct measurement of the mechanical work during translocation by the ribosome. Elife 3: e03406.

[25] Subramaniam AR, Zid BM, O'Shea EK (2014) An integrated approach reveals regulatory controls on bacterial translation elongation. Cell 159: 1200-1211.

[26] Andersson SG, Kurland CG (1990) Codon preferences in free-living microorganisms. Microbiol Rev 54: 198-210.

[27] Shah P, Ding Y, Niemczyk M, Kudla G, Plotkin JB (2013) Rate-limiting steps in yeast protein translation. Cell 153: 1589-1601.

[28] Dobrzyński M, Bruggeman FJ (2009) Elongation dynamics shape bursty transcription and translation. Proc Natl Acad Sci U S A 106: 2583-2588.

[29] Cannarozzi G, Cannarrozzi G, Schraudolph NN, Faty M, von Rohr P, et al. (2010) A role for codon order in translation dynamics. Cell 141: 355-367. 
[30] Guimaraes JC, Rocha M, Arkin AP (2014) Transcript level and sequence determinants of protein abundance and noise in Escherichia coli. Nucleic Acids Res 42: 4791-4799.

[31] Mackey MC, Tyran-Kaminska M, Yvinec R (2013) Dynamic behavior of stochastic gene expression models in the presence of bursting. SIAM Journal on Applied Mathematics 73: $1830-1852$.

[32] Gillespie DT (1992) Markov Processes An Introduction for Physical Scientists. Academic Press, Inc.

[33] Shimada T, Yamazaki Y, Tanaka K, Ishihama A (2014) The whole set of constitutive promoters recognized by RNA polymerase RpoD holoenzyme of Escherichia coli. PLoS One 9: e90447.

[34] Jacob F, Monod J (1961) Genetic regulatory mechanisms in the synthesis of proteins. J Mol Biol 3: 318-356.

[35] Lodish H, Berk A, Zipursky SL, Matsudaira P, Baltimore D, et al. (2000) Molecular Cell Biology. New York, NY: W. H. Freeman, 4th edition.

[36] Reynolds N, O'Shaughnessy A, Hendrich B (2013) Transcriptional repressors: multifaceted regulators of gene expression. Development 140: 505-512.

[37] Murphy KF, Adams RM, Wang X, Balázsi G, Collins JJ (2010) Tuning and controlling gene expression noise in synthetic gene networks. Nucleic Acids Research 38: 2712-2726.

[38] Blake WJ, Kærn M, Cantor CR, Collins JJ (2003) Noise in eukaryotic gene expression. Nature 422: 633-637.

[39] Sharon E, Kalma Y, Sharp A, Raveh-Sadka T, Levo M, et al. (2012) Inferring gene regulatory logic from high-throughput measurements of thousands of systematically designed promoters. Nat Biotechnol 30: 521-530.

[40] Elowitz MB, Levine AJ, Siggia ED, Swain PS (2002) Stochastic gene expression in a single cell. Science 297: 1183-1186.

[41] Rosenfeld N, Young JW, Alon U, Swain PS, Elowitz MB (2005) Gene regulation at the single-cell level. Science 307: 1962-1965. 
[42] Xiong W, Ferrell JE Jr (2003) A positive-feedback-based bistable 'memory module' that governs a cell fate decision. Nature 426: 460-465.

[43] Ingolia NT, Murray AW (2007) Positive-feedback loops as a flexible biological module. Curr Biol 17: 668-677.

[44] To TL, Maheshri N (2010) Noise can induce bimodality in positive transcriptional feedback loops without bistability. Science 327: 1142-1145.

[45] Becskei A, Serrano L (2000) Engineering stability in gene networks by autoregulation. Nature 405: 590-593.

[46] Thattai M, van Oudenaarden A (2001) Intrinsic noise in gene regulatory networks. Proceedings of the National Academy of Sciences of the United States of America 98: 8614-8619.

[47] Mantegna RN, Spagnolo B (1996) Noise enhanced stability in an unstable system. Physical review letters 76: 563 .

[48] Priplata A, Niemi J, Salen M, Harry J, Lipsitz LA, et al. (2002) Noise-enhanced human balance control. Physical Review Letters 89: 238101.

[49] Priplata AA, Niemi JB, Harry JD, Lipsitz LA, Collins JJ (2003) Vibrating insoles and balance control in elderly people. Lancet 362: 1123-1124.

[50] D'Odorico P, Laio F, Ridolfi L (2005) Noise-induced stability in dryland plant ecosystems. Proc Natl Acad Sci U S A 102: 10819-10822.

[51] Turcotte M, Garcia-Ojalvo J, Süel GM (2008) A genetic timer through noise-induced stabilization of an unstable state. Proceedings of the National Academy of Sciences 105: 15732-15737.

[52] Zeng C, Wang H (2010) Colored noise enhanced stability in a tumor cell growth system under immune response. Journal of Statistical Physics 141: 889-908.

[53] Arias AM, Hayward P (2006) Filtering transcriptional noise during development: concepts and mechanisms. Nat Rev Genet 7: 34-44.

[54] Oxender DL, Zurawski G, Yanofsky C (1979) Attenuation in the Escherichia coli tryptophan operon: role of RNA secondary structure involving the tryptophan codon region. Proc Natl Acad Sci U S A 76: 5524-5528. 
[55] Yanofsky C, Horn V (1994) Role of regulatory features of the trp operon of Escherichia coli in mediating a response to a nutritional shift. J Bacteriol 176: 6245-6254.

[56] Ingolia NT (2014) Ribosome profiling: new views of translation, from single codons to genome scale. Nat Rev Genet 15: 205-213.

[57] Gibson G, Dworkin I (2004) Uncovering cryptic genetic variation. Nat Rev Genet 5: 681690.

[58] López-Maury L, Marguerat S, Bähler J (2008) Tuning gene expression to changing environments: from rapid responses to evolutionary adaptation. Nat Rev Genet 9: 583-593.

[59] Hill AV (1910) The possible effects of the aggregation of the molecules of hmoglobin on its dissociation curves. The Journal of Physiology 40: i-vii.

[60] Shea MA, Ackers GK (1985) The OR control system of bacteriophage lambda: A physicalchemical model for gene regulation. J Mol Biol 181: 211-230.

[61] McClure WR (1985) Mechanism and control of transcription initiation in prokaryotes. Annu Rev Biochem 54: 171-204.

[62] Ptashne M (1992) A Genetic Switch. Cell Press \& Blackwell Scientific Publishing.

[63] Arkin A, Ross J, McAdams H (1998) Stochastic kinetic analysis of developmental pathway bifurcation in phage lambda-infected Escherichia coli cells. Genetics 149: 1633-1648.

[64] Gertz J, Siggia ED, Cohen BA (2009) Analysis of combinatorial cis-regulation in synthetic and genomic promoters. Nature 457: 215-218.

[65] Blake W, Balázsi G, Kohanski M, Isaacs F, Murphy K, et al. (2006) Phenotypic consequences of promoter-mediated transcriptional noise. Molecular cell 24: 853-865.

[66] Ajo-Franklin CM, Drubin DA, Eskin JA, Gee EPS, Landgraf D, et al. (2007) Rational design of memory in eukaryotic cells. Genes Dev 21: 2271-2276.

[67] Murphy KF, Balàzsi G, Collins JJ (2007) Combinatorial promoter design for engineering noisy gene expression. Proc Natl Acad Sci U S A 104: 12726-12731.

[68] Fordyce PM, Gerber D, Tran D, Zheng J, Li H, et al. (2010) De novo identification and biophysical characterization of transcription-factor binding sites with microfluidic affinity analysis. Nat Biotechnol 28: 970-975. 
[69] Wang X, Kuwahara H, Gao X (2014) Modeling DNA affinity landscape through two-round support vector regression with weighted degree kernels. BMC Syst Biol 8 Suppl 5: S5.

[70] Steege DA (2000) Emerging features of mRNA decay in bacteria. RNA 6: 1079-1090.

[71] Houseley J, Tollervey D (2009) The many pathways of RNA degradation. Cell 136: 763776. 


\section{Figure Legends}

Figure 1 An illustration of the regulation of gene expression considered in our model.

Figure 2 The effects of the protein burst size distribution on the protein abundance. $\mu_{1}=10$, $k_{\text {prod }}=0.4 \mathrm{~min}^{-1}$, and $k_{\text {deg }}=0.02 \mathrm{~min}^{-1}$. The volume of the cell is set to $1.66 \mathrm{fl}$. The left pane shows the protein abundance distribution given different distributions of $V$. The right pane shows quantile-quantile plots of each protein abundance distribution with respect to the gamma distribution $f(v ; \alpha, \beta)$ with the shape parameter $\alpha=k_{p r o d} / k_{d e g}$ and the scale parameter $\beta=\mu_{1}$. (a) Protein burst size following a geometrically distribution with the mean being $\mu_{1}$. (b) A negative binomial distribution, $f(v ; r, m)$, with size parameter $r=2$ and the mean $m=\mu_{1}$.

Figure 3 The prediction of the stable state $x_{s s}$ and the stability width $w$. The heat maps show simulated results for protein concentration distributions with respect to various settings for the distribution of the bursting size, while the points in back with error bars indicate the prediction. The value of $\mu_{1}$ is fixed to be 10 , while the value of $k_{\text {prod }}$ was varied: 5 (the left pane), 20 (the middle pane), and 50 (the right pane). (a)-(c) Bursting size following truncated geometric distributions in which geometric distributions with the mean being $\mu_{1}$ is right-truncated to have various maximum values. (d)-(f) Bursting size following negative binomial distributions, $f\left(v ; \mu_{1}, r\right)$, where the size parameter $r$ is varied. (g)-(i) Bursting size following discrete uniform distributions with $\left[\mu_{1}-a, \mu_{1}+a\right]$ being the interval in which the number of values in the interval is changed. The color in the z-axis is based on $-\sqrt{\log \left(g_{\max } / g(x)\right)}$ where $g_{\max }$ is the value of the maximum frequency and $g(x)$ is the frequency of $x$.

Figure 4 The protein abundance with respect to various distribution of the signal and the bursting size. (a) A schematic of the setting. $S$ and $V$ are negative binomial random variables. (b) The mean of the mRNA synthesis rate as a function of the mean of $S$. Here, $q(s)=k_{b} /\left(1+\left(s / K_{d}\right)^{n}\right)$ where $k_{b}=2.0 \mathrm{~min}^{-1}, K_{d}=50$, and $n=2$. The size parameter of $S$ are set to 1,4 , and 10 . The red curve shows the deterministic $q(s)$. (c) The stable state $x_{s s}$ with respect to various distribution of $S$ and $V$. The mean of $V$ is fixed to be 10, while its size parameter is set to 1 and 10. Red lines represent the deterministic steady state. (d) The coefficient of variation of $X_{\infty}$ calculated from the simulation performed in 
(c). Each simulation result is based on 10,000 stochastic simulation runs. The error bar represents the standard error of the mean.

Figure 5 The effects of changes in $\mu_{2}$ on the protein expression distribution $p_{s}(x)$ in positive feedback-based gene expression processes with various types of binding cooperativity. The parameters are $\mu_{1}=20, k_{a}=0.1 \mathrm{~min}^{-1}, k_{b}=0.02 \mathrm{~min}^{-1}, K_{d}=50, k_{\text {deg }}=0.02 \mathrm{~min}^{-1}$ The volume of the cell is assumed to be $1.66 \mathrm{fl}$.

Figure 6 Changes in stability characteristics of a non-cooperative positive feedback gene expression process with respect to changes in $\mu_{2}$. The red area represents the region where $X(t)$ has a single stable state and no unstable state. The four histogram plots on the right show the prediction from our model as $\mu_{2}$ is varied while $\mu_{1}$ is fixed to 20 .

Figure 7 An illustration of a phenomenon in which an increase in $\mu_{2}$ is predicted to result in a decrease of the variance in $X_{\infty}$ of gene expression processes with positive feedback loop. The parameters are: $\mu_{1}=5, k_{a}=0.49 \mathrm{~min}^{-1}, k_{b}=0.022 \mathrm{~min}^{-1}, K_{d}=50, k_{d e g}=$ $0.02 \mathrm{~min}^{-1}$.

Figure 8 The effects of changes in $\mu_{2}$ on the protein expression distribution $p_{s}(x)$ in negative feedback-based gene expression processes with various types of binding cooperativity. The parameters are $\mu_{1}=20, k_{b}=0.1 \mathrm{~min}^{-1}, K_{d}=50, k_{\text {deg }}=0.02 \mathrm{~min}^{-1}$ The volume of the cell is assumed to be $1.66 \mathrm{fl}$. 


\section{Figures}

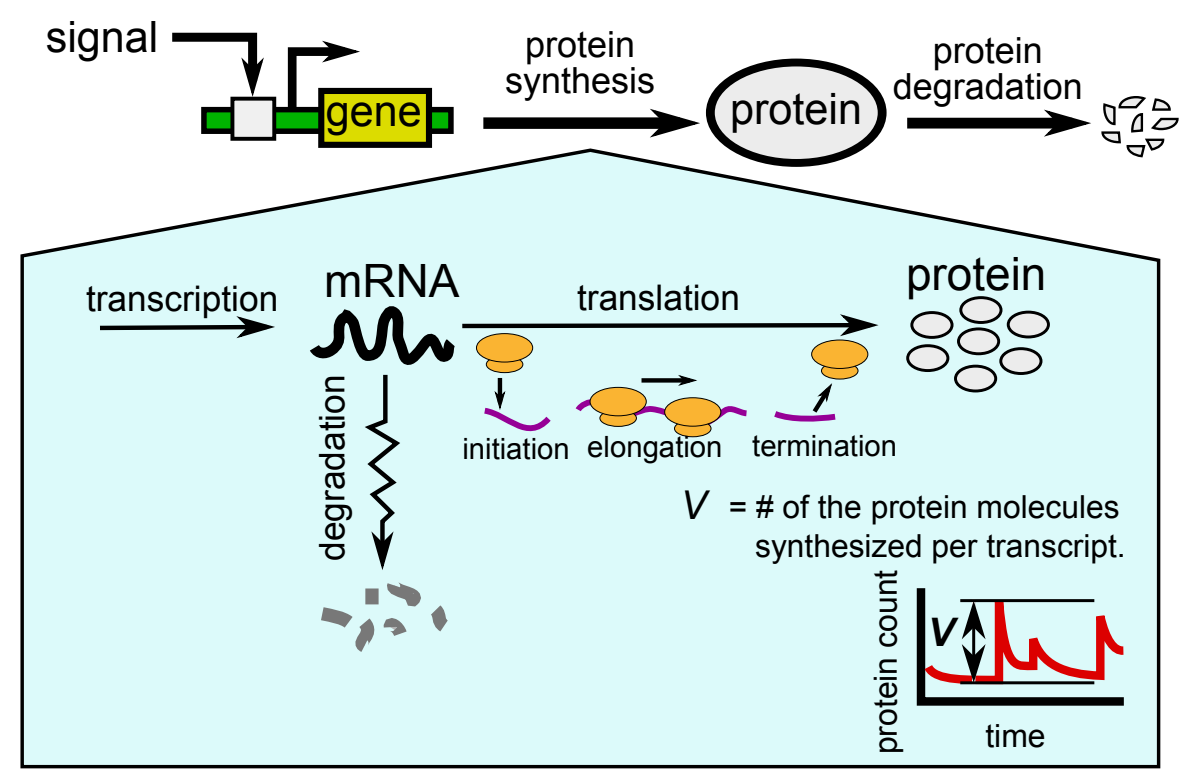

Figure 1. An illustration of the regulation of gene expression considered in our model. 

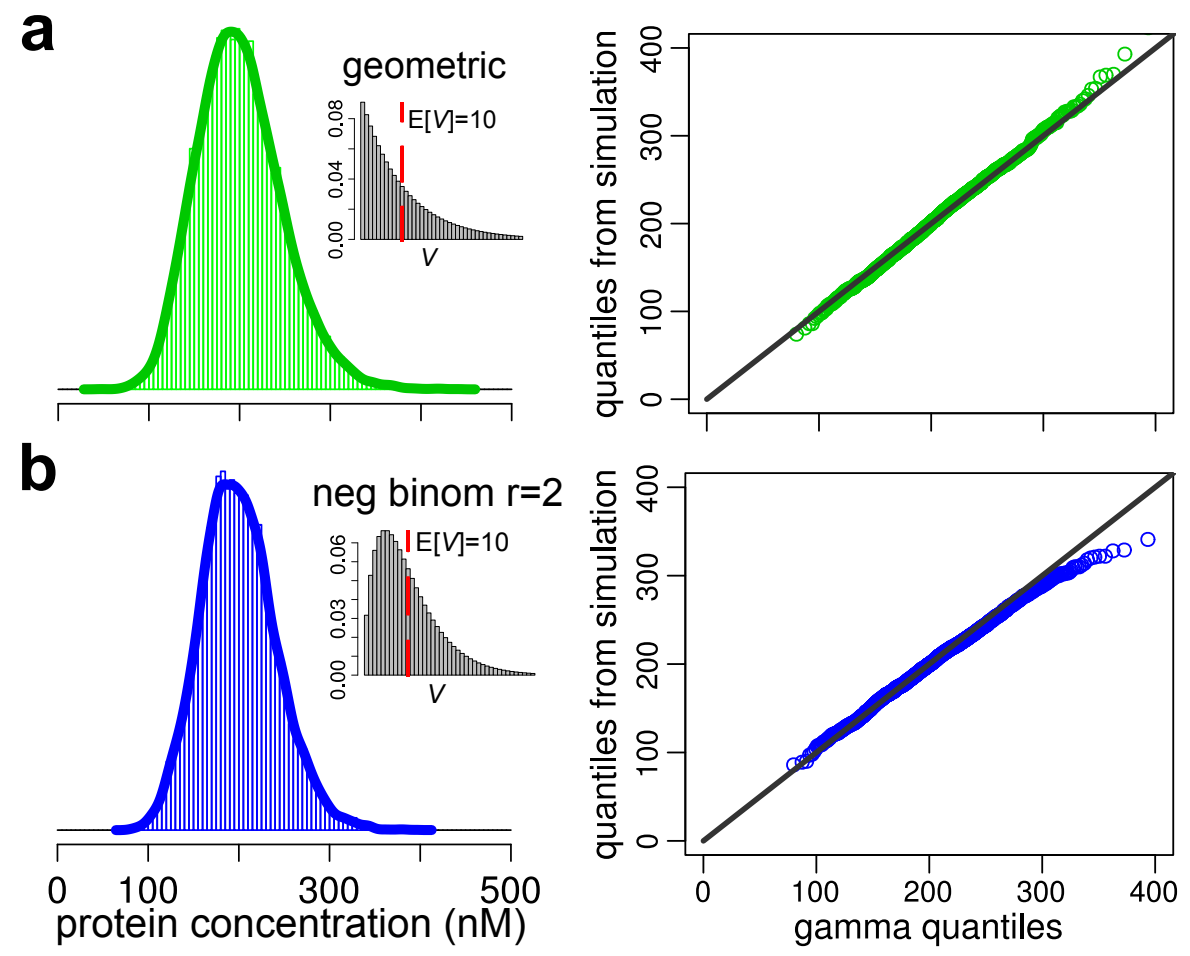

Figure 2. The effects of the protein burst size distribution on the protein abundance. $\mu_{1}=10, k_{\text {prod }}=0.4 \mathrm{~min}^{-1}$, and $k_{\text {deg }}=0.02 \mathrm{~min}^{-1}$. The volume of the cell is set to $1.66 \mathrm{fl}$. The left pane shows the protein abundance distribution given different distributions of $V$. The right pane shows quantile-quantile plots of each protein abundance distribution with respect to the gamma distribution $f(v ; \alpha, \beta)$ with the shape parameter $\alpha=k_{\text {prod }} / k_{\text {deg }}$ and the scale parameter $\beta=\mu_{1}$. (a) Protein burst size following a geometrically distribution with the mean being $\mu_{1}$. (b) A negative binomial distribution, $f(v ; r, m)$, with size parameter $r=2$ and the mean $m=\mu_{1}$. 


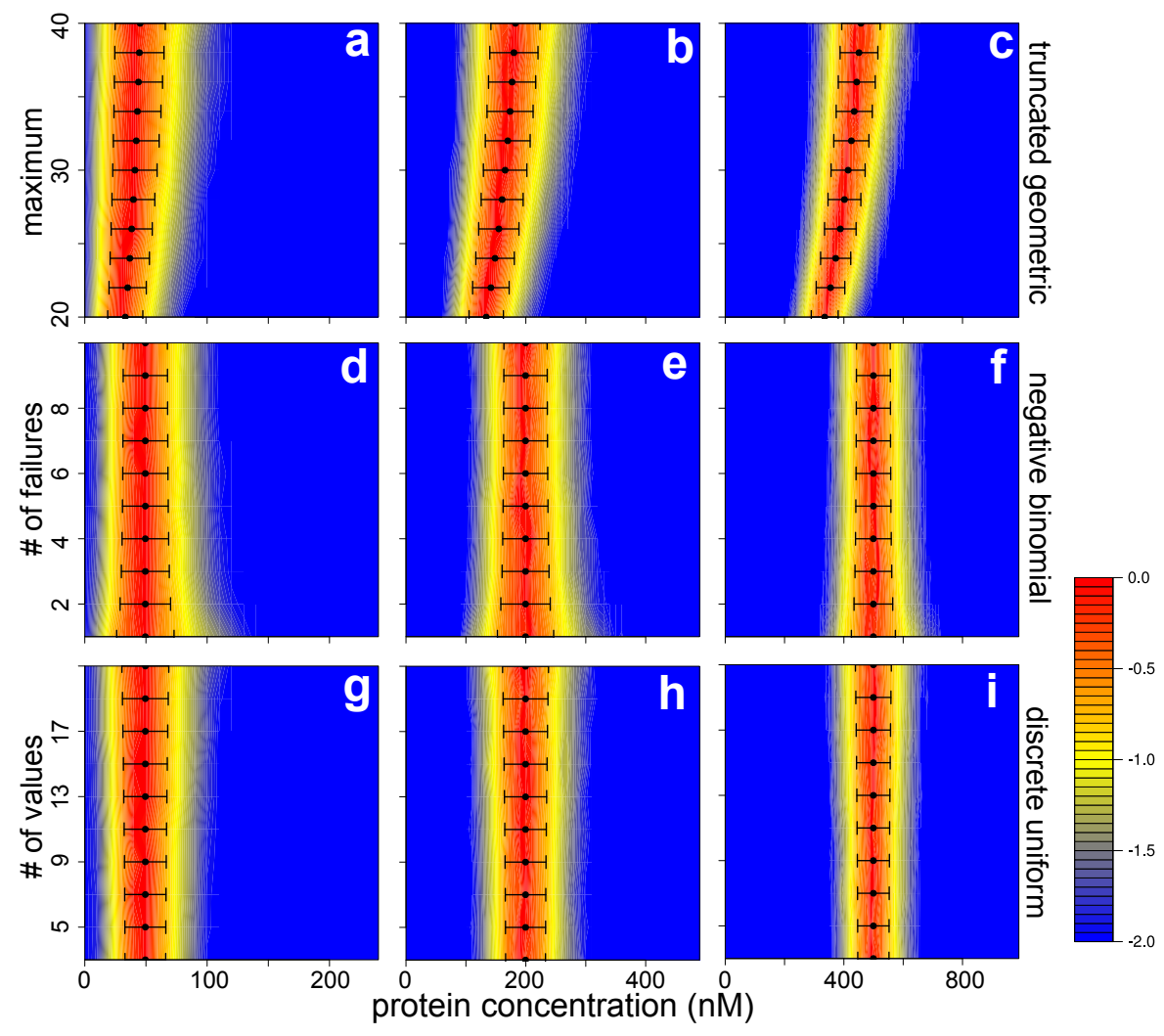

Figure 3. The prediction of the stable state $x_{s s}$ and the stability width $w$. The heat maps show simulated results for protein concentration distributions with respect to various settings for the distribution of the bursting size, while the points in back with error bars indicate the prediction. The value of $\mu_{1}$ is fixed to be 10 , while the value of $k_{\text {prod }}$ was varied: 5 (the left pane), 20 (the middle pane), and 50 (the right pane). (a)-(c) Bursting size following truncated geometric distributions in which geometric distributions with the mean being $\mu_{1}$ is right-truncated to have various maximum values. (d)-(f) Bursting size following negative binomial distributions, $f\left(v ; \mu_{1}, r\right)$, where the size parameter $r$ is varied. (g)-(i) Bursting size following discrete uniform distributions with $\left[\mu_{1}-a, \mu_{1}+a\right]$ being the interval in which the number of values in the interval is changed. The color in the z-axis is based on $-\sqrt{\log \left(g_{\max } / g(x)\right)}$ where $g_{\max }$ is the value of the maximum frequency and $g(x)$ is the frequency of $x$. 
a

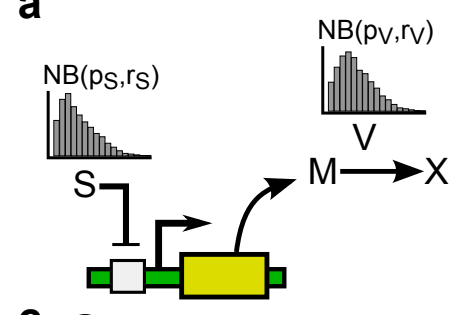

b
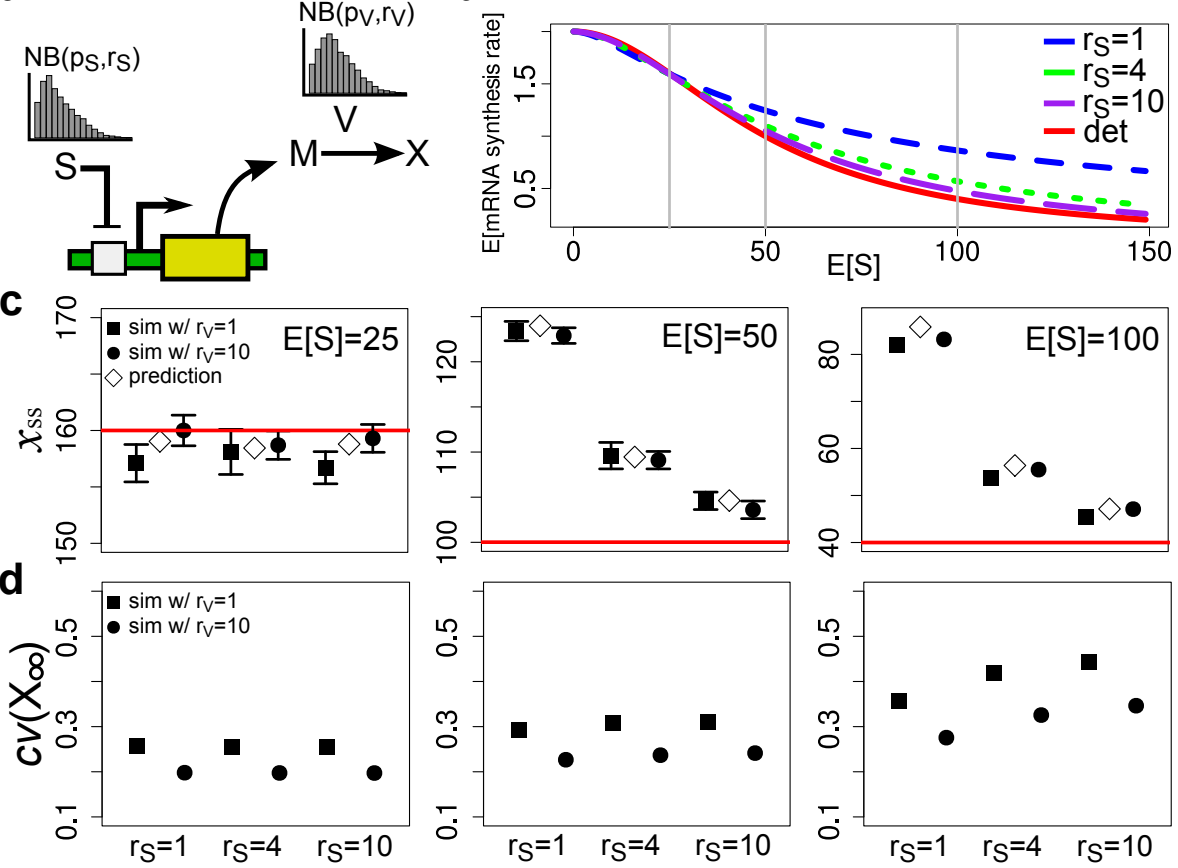

Figure 4. The protein abundance with respect to various distribution of the signal and the bursting size. (a) A schematic of the setting. $S$ and $V$ are negative binomial random variables. (b) The mean of the mRNA synthesis rate as a function of the mean of $S$. Here, $q(s)=k_{b} /\left(1+\left(s / K_{d}\right)^{n}\right)$ where $k_{b}=2.0 \mathrm{~min}^{-1}, K_{d}=50$, and $n=2$. The size parameter of $S$ are set to 1,4 , and 10 . The red curve shows the deterministic $q(s)$. (c) The stable state $x_{s s}$ with respect to various distribution of $S$ and $V$. The mean of $V$ is fixed to be 10, while its size parameter was allowed to change between 1 and 10. Red lines represent the deterministic steady state. (d) The coefficient of variation of $X_{\infty}$ calculated from the simulation performed in (c). Each simulation result is based on 10,000 stochastic simulation runs. The error bar represents the standard error of the mean. 

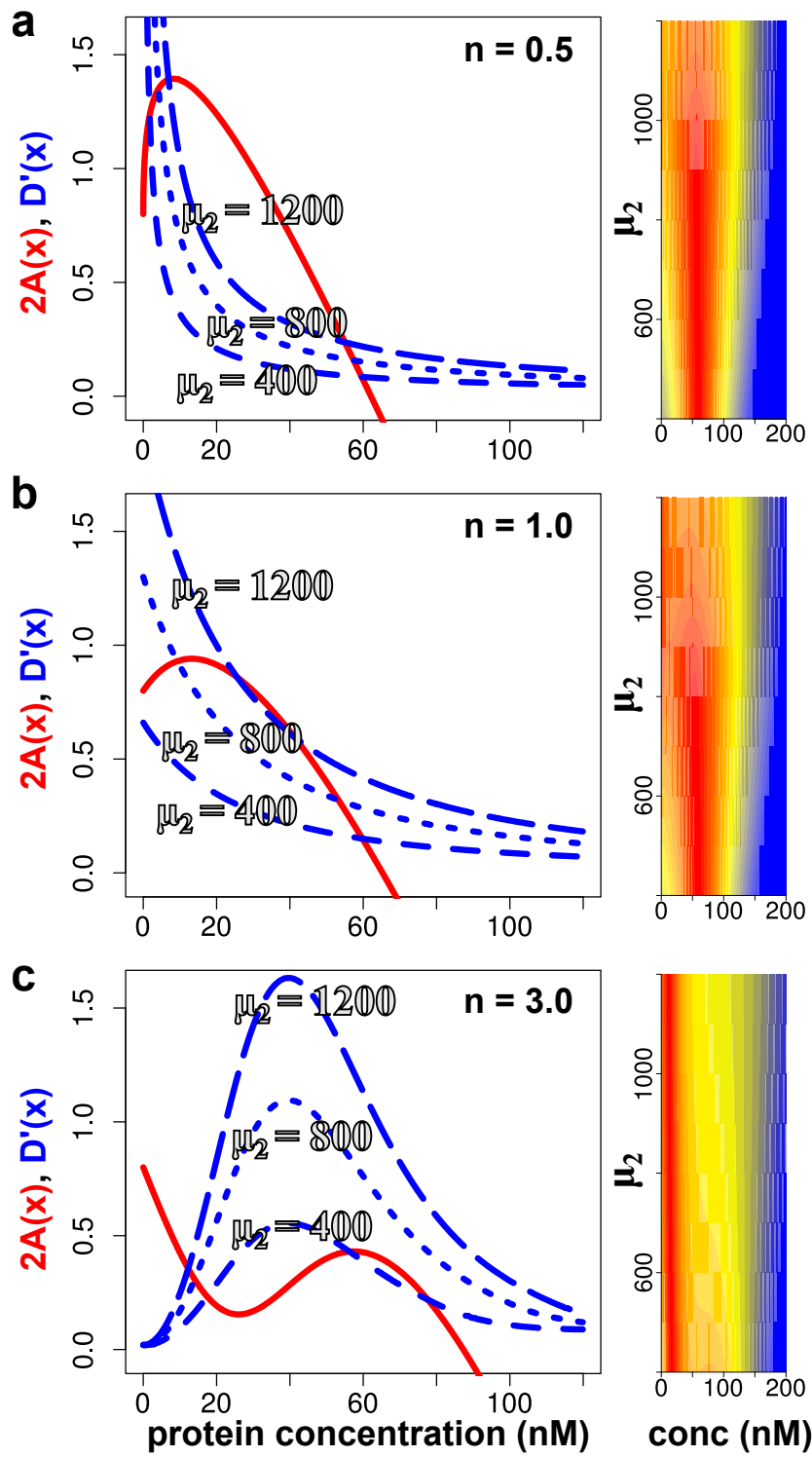

Figure 5. The effects of changes in $\mu_{2}$ on the protein expression distribution $p_{s}(x)$ in positive feedback-based gene expression processes with various types of binding cooperativity. The parameters are $\mu_{1}=20, k_{a}=0.1 \mathrm{~min}^{-1}, k_{b}=0.02 \mathrm{~min}^{-1}, K_{d}=50, k_{d e g}=0.02 \mathrm{~min}^{-1}$ The volume of the cell is assumed to be $1.66 \mathrm{fl}$. 


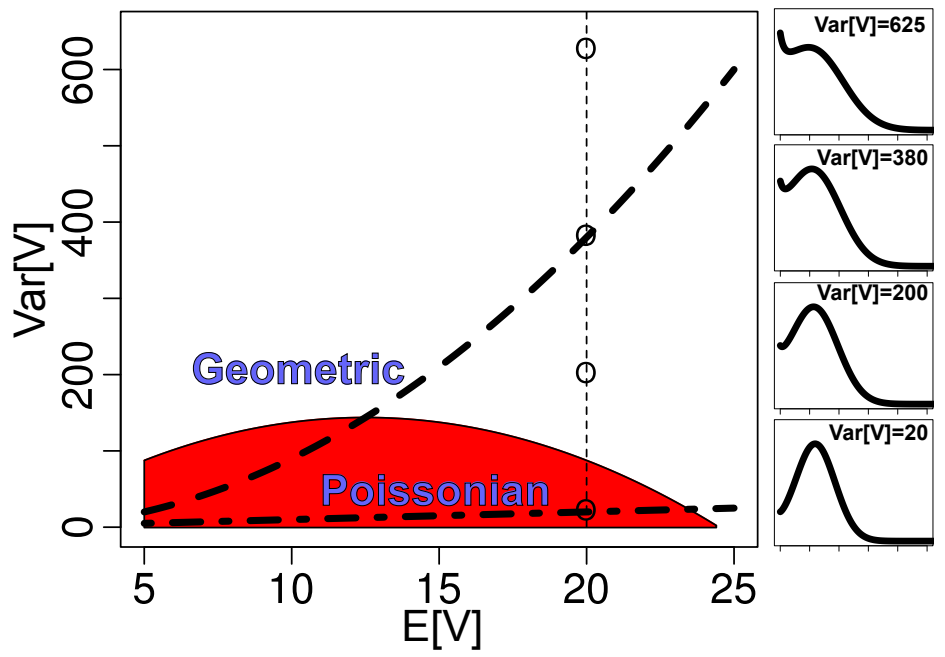

Figure 6. Changes in stability characteristics of a non-cooperative positive feedback gene expression process with respect to changes in $\mu_{2}$. The red area represents the region where $X(t)$ has a single stable state and no unstable state. The four histogram plots on the right show the prediction from our model as $\mu_{2}$ is varied while $\mu_{1}$ is fixed to 20 . 


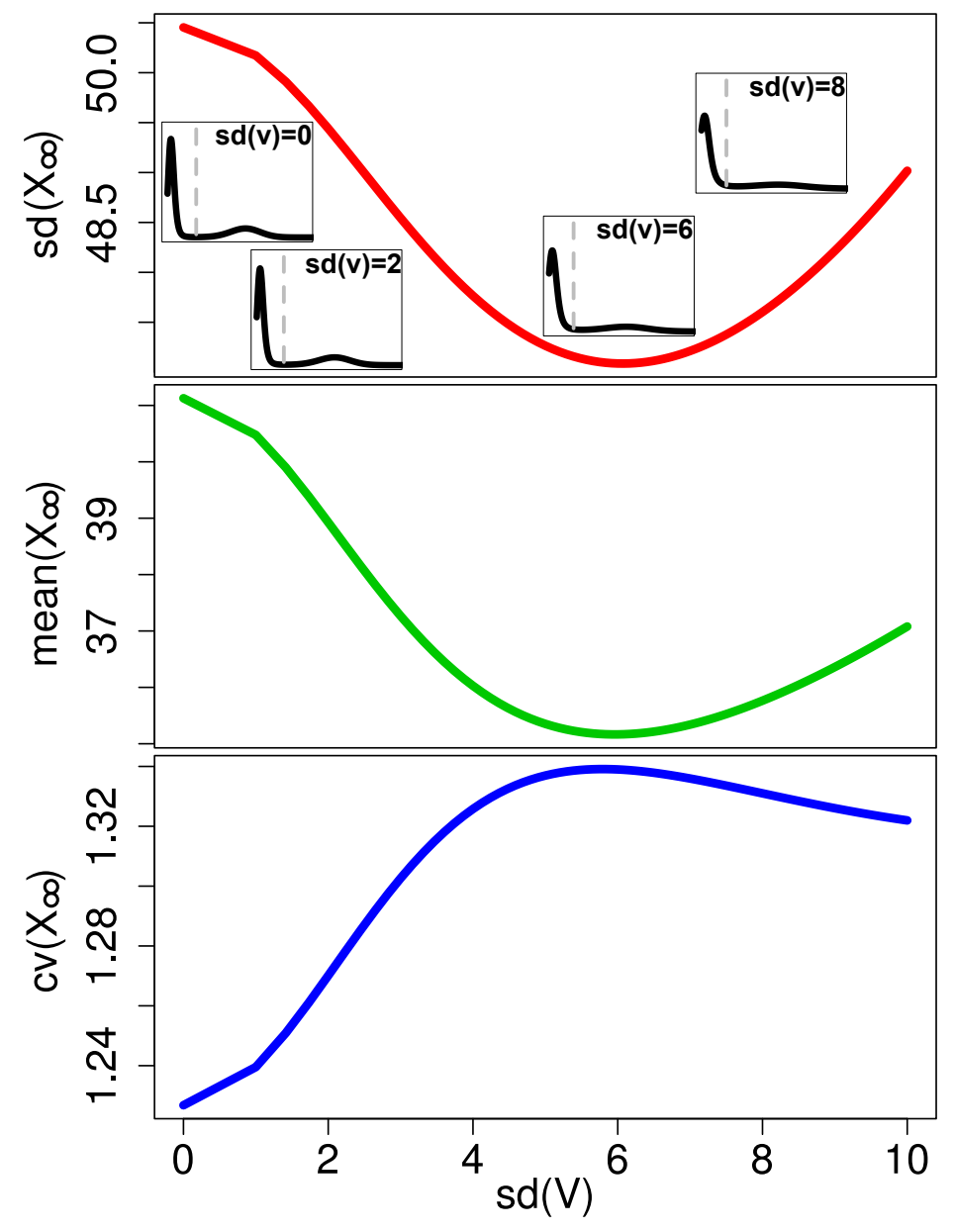

Figure 7. An illustration of a phenomenon in which an increase in $\mu_{2}$ is predicted to result in a decrease of the variance in $X_{\infty}$ of gene expression processes with positive feedback loop.

The parameters are: $\mu_{1}=5, k_{a}=0.49 \mathrm{~min}^{-1}, k_{b}=0.022 \mathrm{~min}^{-1}, K_{d}=50, k_{\text {deg }}=0.02 \mathrm{~min}^{-1}$. 

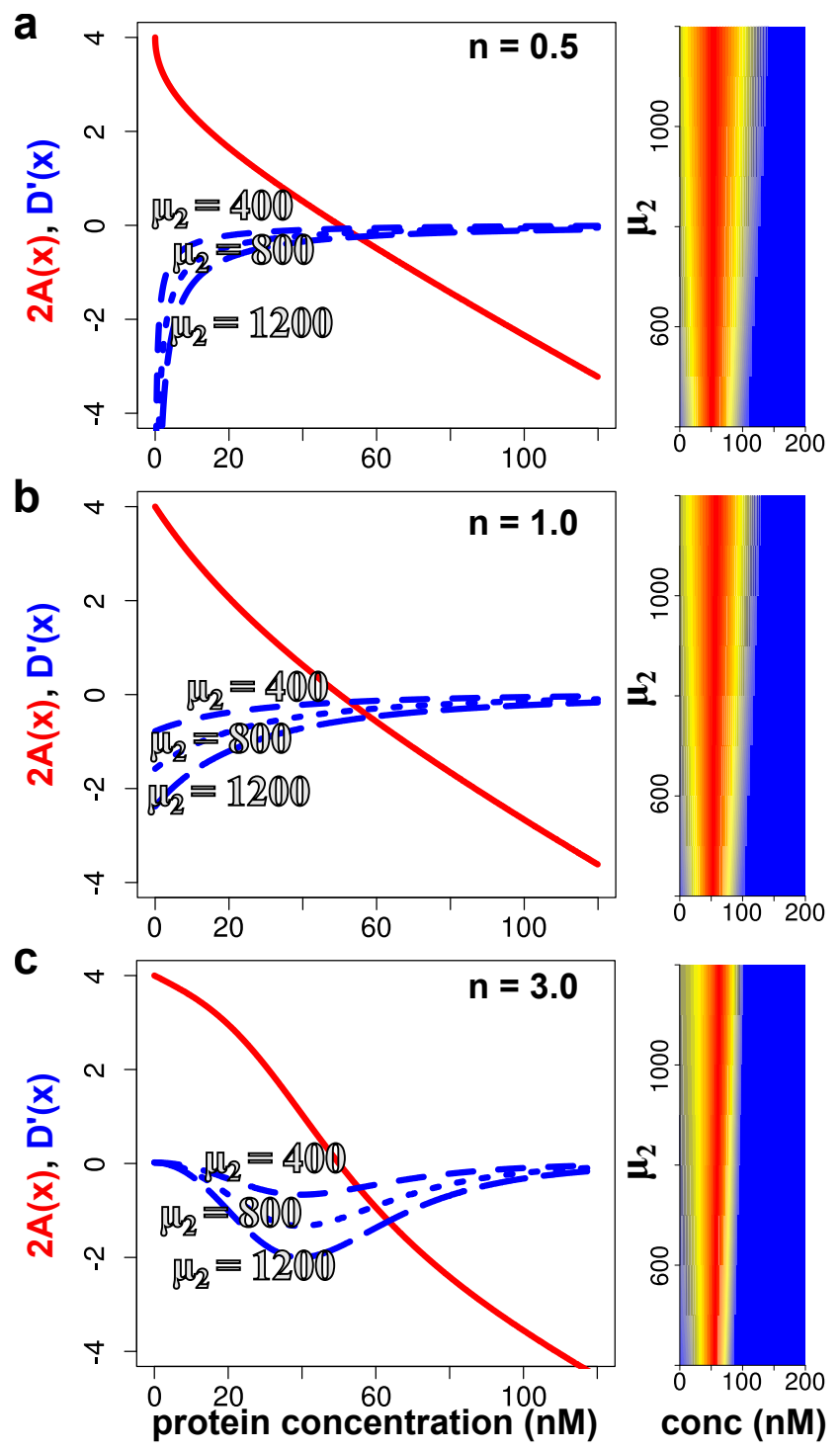

Figure 8. The effects of changes in $\mu_{2}$ on the protein expression distribution $p_{s}(x)$ in negative feedback-based gene expression processes with various types of binding cooperativity. The parameters are $\mu_{1}=20, k_{b}=0.1 \mathrm{~min}^{-1}, K_{d}=50, k_{d e g}=0.02 \mathrm{~min}^{-1}$ The volume of the cell is assumed to be $1.66 \mathrm{fl}$. 